\title{
Fisura cervical media. Reporte de un caso
}

\author{
ELIZABETH REMOLCOIS C. ${ }^{2}$, PABLO LARRAGUIBEL Q. ${ }^{2}$, \\ GABRIELA LÓPEZ V. ${ }^{1}$, JOSÉ ANTONIO SEPÚLVEDA C. ${ }^{3}$ \\ 1. Alumna Medicina $5^{\circ}$ año UDD, Santiago. \\ 2. Alumnos Medicina $5^{\circ}$ año Universidad Austral, Valdivia. \\ 3. Jefe Unidad Cirugía y Ortopedia Infantil Hospital Clínico Magallanes, Punta Arenas.
}

\begin{abstract}
\section{Congenital mid-line cervical cleft: a case report}

Introduction: Congenital midline cervical cleft is a rare congenital mid-line anomaly of the neck. There are different theories as to the origins of CMCC. Nevertheless, most believe that is due to impaired fusion of the first and second branchial arches during mesoderm formation. There are less than 100 reported cases in the literature. Case report: A newborn with a mid-line cervical injury without other associated abnormalities. A complete excision of the lesion and the blind-ended sinus were performed and the skin was closed with a Z-plasty approach. Discussion: Congenital mid-line cervical cleft requires early diagnosis, the study of associated abnormalities and an early repair is recommended to aesthetically handle the case and to prevent blind-ended sinus infection.
\end{abstract}

(Key words: Congenital malformations of the neck, cervical cleft).

Rev Chil Pediatr 2013; 84 (1): 80-82

\section{RESUMEN}

Introducción: La fisura cervical media o cursiva cervical es una anomalía congénita rara de la línea media. Existen varias hipótesis para explicar su origen, la más aceptada es la falla en la fusión del primer y segundo arco branquial durante la mesodermización. Han sido reportados menos de 100 casos en la literatura. Caso clínico: Se reporta el caso de un recién nacido con una lesión cervical media sin otras malformaciones asociadas. Se realizó extracción completa de la lesión visible y del seno ciego caudal, cerrándose el defecto con una Zplastía. Discusión: La fisura cervical media requiere de un diagnóstico precoz y un estudio de malformaciones asociadas, junto con una resolución temprana de este defecto, tanto para el manejo estético como para evitar retracciones e infección del seno ciego.

(Palabras clave: Malformaciones congénitas de cuello, fisura cervical).

Rev Chil Pediatr 2013; 84 (1): 80-82

Recibido el 11 de diciembre de 2011, devuelto para corregir el 16 de febrero de 2012, segunda versión el 29 de julio de 2012 , aceptado para publicación el 29 de septiembre de 2012.

Este trabajo cumple con los requisitos sobre consentimiento /asentimiento informado, comité de ética, financiamiento, estudios animales y sobre la ausencia de conflictos de intereses según corresponda.

Correspondencia a:

José Antonio Sepúlveda C.

E-mail: drpeposepulvedachile@yahoo.com 


\section{Introducción}

La fisura cervical media consiste en una desepitelización cutánea con una protuberancia fibromatosa en su extremo proximal que se extiende hacia caudal y termina en un seno ciego, que se dirige hacia la horquilla o hueco esternal $^{2}$. Existen varias hipótesis para explicar su origen. La más aceptada es la falla en la fusión del primer y segundo arco branquial durante la mesodermización. Otras hipótesis incluyen la exteriorización de un remanente del conducto tirogloso o el aumento de la presión en el área cervical desde el techo pericárdico en el embrión en desarrollo ${ }^{3}$.

Aunque es una malformación bastante característica, sus principales diagnósticos diferenciales son las fístulas y senos de origen branquial y los quistes broncogénicos. La patología branquial (quiste, seno o fístula) suele ubicarse más lateralmente en el cuello, por ventral al músculo esternocleidomastoideo, aunque apenas desviada, una fístula así puede confundirse. Por otra parte, los quistes broncogénicos están ubicados habitualmente en el hueco supraesternal con posible extensión hacia el mediastino. Para realizar correctamente el diagnóstico, se suele recurrir a exámenes imagenológicos como la ecografía o la tomografía axial computarizada ${ }^{4}$.

Se ha demostrado asociación con otras malformaciones congénitas: quiste tirogloso, fisura labial inferior, de la lengua, de la mandíbula, espolón mandibular, microgenia, ectopia cordis torácica y otras malformaciones cardíacas e hipoplasia de otras estructuras de la línea media ${ }^{2,3,5}$.

El objetivo de esta comunicación es presentar el caso de un recién nacido portador de una infrecuente malformación como es la fisura cervical media.

\section{Caso clínico}

Paciente de sexo masculino recién nacido de la maternidad del Hospital Clínico de Magallanes en enero de 2011, sin antecedentes relevantes del embarazo, que presentaba una lesión en la línea media cervical a $2 \mathrm{~cm}$ sobre el hueco esternal cuyas dimensiones eran de 3 cm de largo x 0,5 cm de ancho. En su extremo proximal se observaba una protuberancia de tejido y en su extremo caudal, un seno ciego (figura 1).

Clínicamente la lesión no se encontraba adherida a planos profundos y no presentaba movilidad con la protrusión de la lengua o la deglución. El examen físico del resto de la cara, cuello y cavidad oral no arrojó alteraciones.

Para completar el estudio se realizó una ecografía de cuello, un ecocardiograma y una radiografía de tórax, con el objeto de descartar, por una parte los otros diagnósticos diferenciales de lesiones cervicales medias y por otro lado las malformaciones asociadas. Los exámenes no revelaron alteraciones.

En la cirugía se realizó la extracción completa de la lesión visible (figura 2), disección y extirpación del seno que se extendía $2 \mathrm{~cm}$ ha-

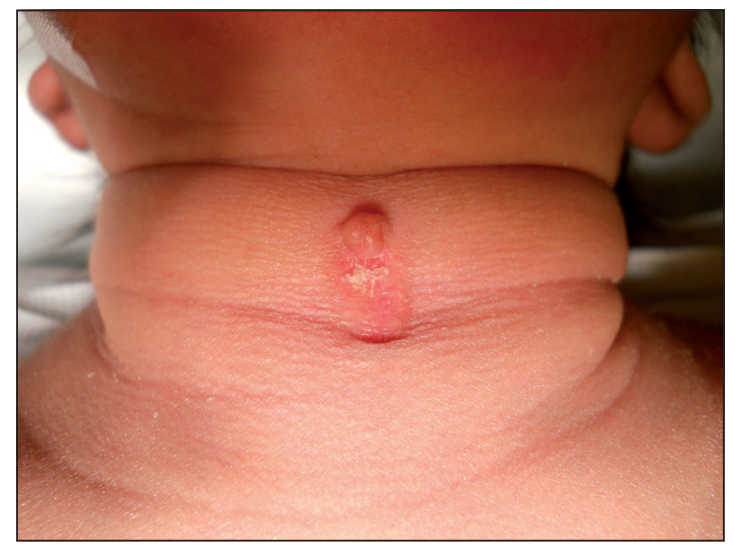

Figura 1. Fisura cervical media en recién nacido.

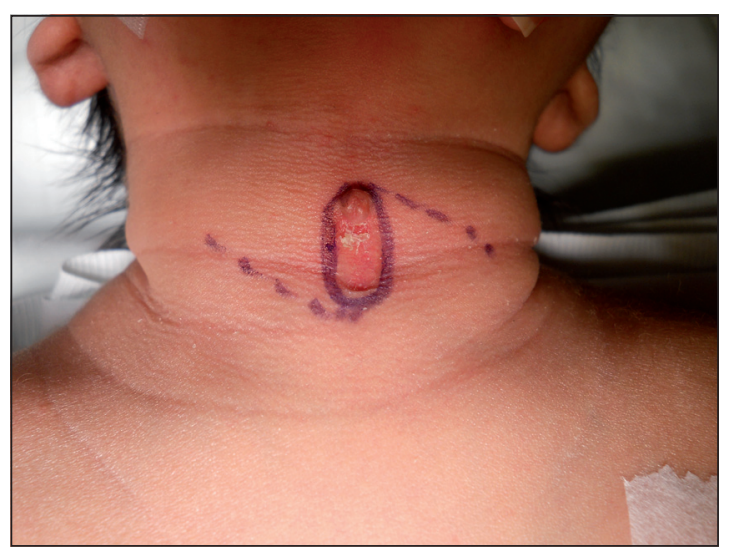

Figura 2. Planificación quirúrgica, previa a la extirpación del seno. 


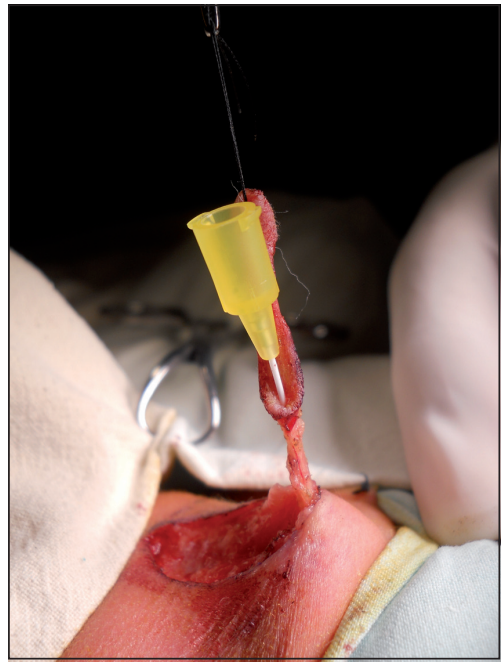

Figura 3. Disección de seno ciego.

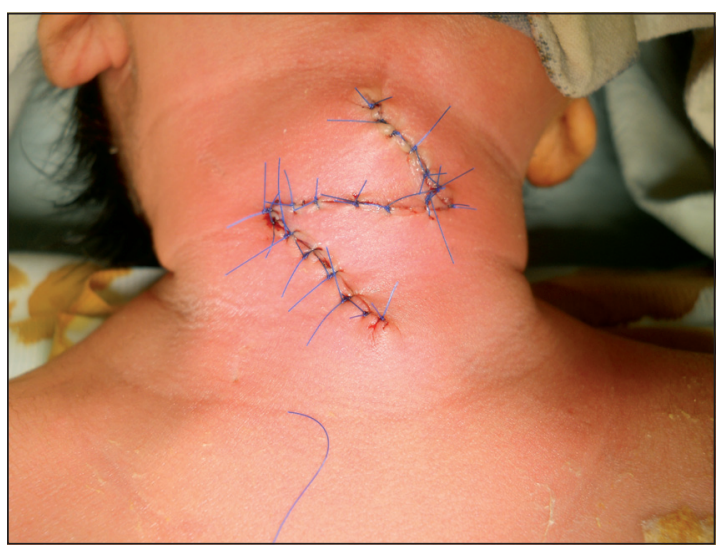

Figura 4. Z-plastía, visión postoperatorio.

cia caudal y terminaba en fondo de saco (figura 3). Se cerró el defecto con una Z-plastía de piel y tejido subcutáneo (figura 4).

\section{Discusión}

La fisura cervical es una rara malformación de la línea media cervical, que puede confundirse fácilmente con otras más frecuentes como las fístulas y senos de origen branquial. A veces puede ser difícil diagnosticar esta lesión por no presentarse de forma muy evidente, sin embargo, en este caso la forma de presentación permitió el diagnóstico precoz. Al ser una falla estructural anatómica, la única solución es la cirugía. Ésta cobra especial relevancia ya que, la resolución temprana del defecto como en este caso clínico, permite evitar la retracción e infección del seno ciego, como también la corrección estética antes que el menor tenga conciencia de ello y pueda ser objeto de problemas de tipo psicológico. El tratamiento quirúrgico de elección, $\mathrm{y}$ usado por el equipo médico tratante, es la extirpación y reparación con colgajos en $\mathrm{Z}$. Se recomienda el seguimiento de mediano plazo para evaluar resultado estético final y evitar retracciones y cicatrices hipertróficas.

\section{Referencias}

1.- Agrawal V, Sanghvi B, Borwankar S: Congenital Midline Cervical Cleft: A Case Report. The Internet Journal of Surgery. 2010; 22, no1. Disponible en: http://www. ispub.com/journal/the_internet_journal_of_surgery/ volume 22 number_1_1/article/congenital-midline-cervical-cleft-a-case-report.html, última visita 28/07/2011.

2.- Sannajust JP, Barthelemy I, Emering C, Mandard I, Mondie JM: Fente cervicale médiane congénitale. Rev Stomatol Chir Maxillofac 2004; 105: 338-41.

3.- Mlynarek A, Hagr A, Tewfik TL, Nguyen VH: Congenital mid-line cervical cleft: case report and review of literature. Int J Pediatr Otorhinolaryngol 2003; 67 (11): 1243-9.

4.- Tewfik T: Congenital Malformations, Neck. 2010. Disponible en: http://emedicine.medscape.com/ article/837477-overview , última visita 28/07/2011.

5.- Morovic CG, Vidal C: Fisura media cervical. Rev Chil Pediat 1999; 70 (4): 311-3. Disponible en: http://www. scielo.cl/scielo. 